\title{
Successful treatment of catatonic syndrome in bipolar I disorder adding aripiprazole to ECT: A case report
}

\author{
Diego Hidalgo, MD \\ Eduard Parellada, MD, PhD \\ Rocío Martín-Santos, MD, PhD \\ Antonio Benabarre, MD, PhD
}

Servicio de Psiquiatría Adultos, Instituto Clínico de Neurociencias, Hospital Clínic, IDIBAPS, CIBERSAM, Universidad de Barcelona, Barcelona

\section{SPAIN}

ABSTRACT - Background and Objectives: Catatonic syndrome is a condition presenting in multiple ways, sharing many of them with the neuroleptic malignant syndrome and other diseases. This diagnostic challenge is the main cause of keep treating catatonic syndromes without neuroleptics.

Methods: Review of the literature and a case report.

Results: We present the case of a 19 years old bipolar I patient with a severe catatonic syndrome, with a torpid clinical evolution, partial response to benzodiazepines and ECT, which successfully resolved with intramuscular aripiprazole. We found through a systematic review (PubMed 2005-2010) that there are few but significant case reports of catatonic syndromes treated with new second generation antipsychotics for different reasons with good outcomes as ours. The pharmacological profile of aripiprazole and the low incidence of NMS reported make it a suitable option in treating this syndrome.

Conclusions: We think that this case report could contribute to add more evidence for aripiprazole to be considered a good third-line option in the treatment of catatonic syndrome. However, this would require randomized controlled trials to confirm its effectiveness and safety.

Received: 26 April 2011

Revised: 16 December 2011

Accepted: 21 December 2011 


\section{Introduction}

Catatonic syndrome is a life threatening condition, classically described in schizophrenia but could be the expression of many psychiatric and non-psychiatric (infectious, metabolic, neurological, and toxic) diseases. Its presentation is always polymorph with a wide range of signs and symptoms, which make it sometimes difficult to differentiate from other diseases ${ }^{1,2}$. It is probably produced by a complex imbalance of dopamine, serotoninergic, noradrenergic and cholinergic systems at basal ganglia and prefrontal cortex, although the exact mechanism remains unknown ${ }^{1,3,4}$. Catatonic syndrome treatment should be fast, safe and effective. Even though benzodiazepines resolve most of the cases, there are still some of them that are even resistant to electroconvulsive therapy $(\mathrm{ECT})^{5,6}$. The use of first generation antipsychotics showed an important incidence of catatonia worsening and inducing of neurological malignant syndrome (NMS) ${ }^{1,7}$. Nevertheless, the experience with some second generation antipsychotics showed a lower number of cases and even possible beneficial effects in brain function and on the catatonic symptoms ${ }^{8}$. In the last years we found few reported cases which responded to the treatment with aripiprazole when benzodiazepines and ECT failed or when the last was not available ${ }^{9-13}$ (Table 1). Aripiprazole is a second generation antipsychotic with a mechanism of action $\left(\mathrm{D}_{2} / 5\right.$ $\mathrm{HT} 1_{\mathrm{A}}$ partial agonist and 5-HT1 $1_{2 \mathrm{~A}}$ antagonist) that seems suitable for the treatment misbalance neurochemistry that probably produces the catatonic syndrome. Since some theories points that the catatonic syndromes could be explained by a prefrontal hypodopaminergic state, the partial agonism of aripiprazole and rise in dopamine levels in these regions could explain its therapeutic

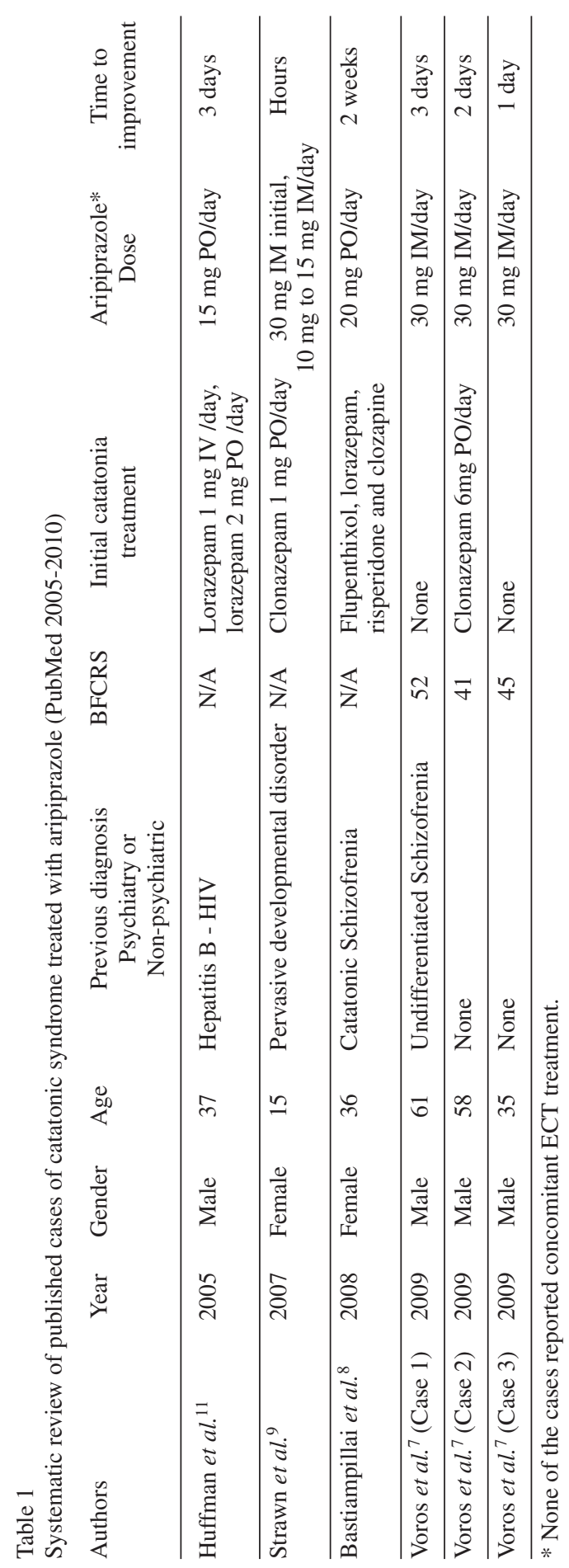


benefits. However, there is not enough evidence that demonstrates its clinical effectiveness (9). We present in this report the case of a bipolar I patient with a severe catatonic syndrome, with a torpid clinical evolution, partial response to benzodiazepines and ECT, which successfully resolved with intramuscular aripiprazole.

Methods: Review of the literature and a case report.

\section{Case presentation}

The case is about a 19 years old Caucasian man, with a well-documented history of type I bipolar disorder by the DSM-IV criteria treated with lithium carbonate and risperidone presenting to the emergency room with grossly disorganized behaviour, disinhibition, agitation and stereotyped movements which required physical restraint and haloperidol $5 \mathrm{mg}$ IM. The family reported a week of expansivity; altered sleep pattern and probable medication drop out, scoring at admission a Young Mania Rating Scale (YMRS) of 56. A couple of hours after admission the patient progressed to mutism, motor rigidity, stupor and fever of $38.9^{\circ} \mathrm{C}$, together with a leukocitosis $\left(14300 / \mathrm{mm}^{3}\right)$ and elevated CPK (2121 U/L) totalizing a BushFrancis Catatonia Rating Scale (BFCRS) of 36. The urine and the rest of blood tests did not show any other abnormal values. He was started on increasing doses of flunitrazepam IM up to $3 \mathrm{mg} /$ day (since parenteral lorazepam is not available in our market and the patient was unable to tolerate oral administration), acetaminophen $1 \mathrm{~g}$ qid, IV fluid therapy and re-started lithium carbonate PO $1200 \mathrm{mg} /$ day. A chest X-ray did not demonstrate any considerable abnormalities and a brain magnetic resonance ex- ploration showed no brain lesions. The clinical picture of the patient became an interesting diagnostic challenge between catatonia, neuroleptic malignant syndrome (NMS), antipsychotic-induced rhabdomyolysis (RML) or an acute infectious disease. After stopping neuroleptics and adding empiric antibiotic coverage, the fever persisted, CPK increased (7408 U/L), and also the rigidity. In front of the clinical situation ECT every other day was indicated, with poor results after the first 4 sessions. The adverse clinical course obligated us to consider other options; we decided to add aripiprazole $15 \mathrm{mg}$ IM/day to the treatment along with ECT at the same parameters and benzodiazepines also at same doses. In the following days the patient showed a continuum clinical progress without fever peaks, being able of doing autonomous basic tasks in 4 days (BFCRS: 16; $44.4 \%$ improvement) and progressive normalization of laboratory values (Leukocytes and CPK). The patient was discharged from our hospital almost 1 month after the admission with IM aripiprazol for two weeks rotated after to PO aripiprazol $30 \mathrm{mg} /$ day for other two weeks along with decreasing doses of flunitrazepam and totalizing 16 ECT sessions showing a full clinical recovery.

\section{Discussion}

Catatonic syndrome could be a challenging diagnosis when it present with severe signs and symptoms as our case ${ }^{1}$. This and the fact that the patient had a history of using antipsychotic medication at home and also the one administered in the emergency room, made things even more difficult to differentiate from an incipient NMS, iatrogenic catatonia, RML or catatonia due to mania. Even though antipsychotics are fre- 
quently associated with rhabdomyolisis, we discard the diagnosis because there were no muscular signs nor myoglobinuria in urine test and normal creatinine-BUN levels in blood tests ${ }^{14}$. At the same time, there was a history of progressive symptoms the previous days that made us think of a catatonia due to mania or induced by the risperidone. Although the acute exacerbation of symptoms and signs along with the elevation of CPK after the administration of IM Haloperidol inclined us to suspect an incipient NMS or an iatrogenic catatonia. There is still an on-going debate if NMS is a subtype of catatonia or not, what we know for sure is that catatonia is a risk factor for NMS. It is also obvious that both share symptomatology and probably neurochemical mechanisms ${ }^{4}$. We consider here the condition as a syndrome as it was impossible to differentiate a specific type or cause. There is a good rate of effectiveness in catatonic patients with benzodiazepines (aprox.70\%), most of them improve in the first four days ${ }^{6}$. ECT usually resolves catatonic syndromes in approximately $90 \%$ of cases, most of them with a substantial improvement within the first two to five sessions ${ }^{15}$. After 6 days of treatment with benzodiazepines and 4 ECT sessions, the patient condition did not seem to improve. The use of second generation antipsychotics in this setting seemed an option, considering the low incidence of adverse effects and the good response in some reported cases ${ }^{9-13}$. Among them, we decided to introduce aripiprazole because it's pharmacological profile and also the low incidence of NMS reported with its use in mania $^{16,17}$ along with the suitable mechanism of action mentioned. Even though we think that benzodiazepines and ECT contributed to the improvement, there was a favourable inflection point in the clinical course with the introduction of aripiprazole.

\section{Conclusion}

Catatonic syndrome is a condition presenting in multiple ways, sharing many of them with the neuroleptic malignant syndrome and other diseases. This diagnostic challenge is the main cause of keep treating catatonic syndromes without neuroleptics. There are few but significant case reports of catatonic syndromes treated with new second generation antipsychotics for different reasons with good outcomes as ours. This fact and taking into account the low risk of NMS reported of these new antipsychotics, may make aripiprazole a good third-line option to be considered in the treatment of catatonic syndrome. However, this would require randomized controlled trials to confirm its effectiveness and safety.

\section{Financial disclosure}

This research received no specific grant from any funding agency in the public, commercial, or not-for-profit sectors.

\section{References}

1. Fink M, Taylor MA. Catatonia: A Clinician's Guide to Diagnosis and Treatment. Cambridge: Cambridge University Press; 2003.

2. Bernardo M, Parellada E, Palao D. Un paciente catatónico. Medicina Integral 1991; 18: 239-244.

3. Lee JWY. Laboratory findings in Catatonia. In: Caroff SN, Mann SC, Francis A, Fricchione GL, editors. Catatonia: from psychopathology to neurobiology. Washington DC: American Psychiatric Publishing; 2004. p. 65-75.

4. Northof G. Catatonia and neuroleptic malignant syndrome: psychopathology and pathophysiology. J Neural Transm 2002; 109: 1453-1467. 
5. Bush G, Fink M, Petrides G, Dowling F, Francis A. Catatonia. II. Treatment with lorazepam and electroconvulsive therapy. Acta Psychiatr Scand 1996; 93: 137-143.

6. Lee JWY, Schwartz DL, Hallmayer J. Catatonia in a Psychiatric Intensive Care Facility: Incidence and Response to Benzodiazepines. Ann Clin Psychiatry 2000; 6: 89-96.

7. Blumer D. Catatonia and the neuroleptics: Psychobiologic significance of remote and recent findings. Compr Psychiatry 1997; 38: 193-201.

8. Van Den Eede F, Van Hecke J, Van Dalfsen A, Van den Bossche B, Cosyns P, Sabbe B. The use of atypical antipsychotics in the treatment of catatonia. Eur Psychiatry $2005 ; 20: 422-429$.

9. Voros V, Kovacs A, Herold R, Osvath P, Simon M, Fekete $\mathrm{S}$, et al. Effectiveness of intramuscular aripiprazole injection in patients with catatonia: report on three cases. Pharmacopsychiatry 2009; 42: 286-287.

10. Bastiampillai T, Dhillon R. Catatonia resolution and aripiprazole. Aust N Zealand J Psychiatry 2008; 42(10): 907.

11. Strawn JR, Delgado SV. Successful treatment of catatonia with aripiprazole in an adolescent with psychosis. J Child Adolesc Psychopharmacol 2007; 17: 733-735.

12. Kirino E. Prolonged Catatonic Stupor Successfully Treated with Aripiprazole in an Adolescent Male with Schizophrenia: A Case Report. Clin Schizophr Relat Psychoses 2010; 4: 1-4.

13. Huffman JC, Fricchione GL. Catatonia and psychosis in a patient with AIDS: treatment with lorazepam and aripiprazole. J Clin Psychopharmacol 2005; 25: 508-510.
14. Melli G, Chaudhry V, Cornblath DR. Rhabdomyolysis: an evaluation of 475 hospitalized patients. Medicine (Baltimore) 2005; 84(6): 377-385.

15. Fink M. Catatonia: syndrome or schizophrenia subtype? Recognition and treatment. J Neural Transm 2001; 108: 637-644.

16. Keck PE, Marcus R, Tourkodimitris S, Ali M, Liebeskind A, Saha A, et al. A placebo-controlled, doubleblind study of the efficacy and safety of aripiprazole in patients with acute bipolar mania. Am J Psychiatry 2003; 160(9): 1651-1658.

17. Vieta E, Bourin M, Sanchez R, Marcus R, Stock E, McQuade R, et al. Effectiveness of aripiprazole vs. haloperidol in acute bipolar mania. Br J Psychiatry 2005; 187: 235-242.

Author for correspondence:

Diego Hidalgo

Servicio de Psiquiatría Adultos

Instituto Clínico de Neurociencias

Hospital Clínic, Villarroel n ${ }^{\circ}$ 170,

08036, Barcelona, Spain

Phone: +34932 275400

Fax: +34 932275548

E-mail: dahidalg@clinic.ub.es 